\title{
Review Article \\ Involvement of Activating NK Cell Receptors and Their Modulation in Pathogen Immunity
}

\author{
Francesco Marras, ${ }^{1}$ Federica Bozzano, ${ }^{2}$ and Andrea De Mariaa ${ }^{1,3,4}$ \\ ${ }^{1}$ Centro di Eccellenza per la Ricerca Biomedica (CEBR), Università di Genova, 16132 Genova, Italy \\ ${ }^{2}$ Istituto G. Gaslini, 16147 Genova, Italy \\ ${ }^{3}$ Department of Health Sciences (DISSAL), University of Genova, 13132 Genova, Italy \\ ${ }^{4}$ Infectious Diseases Unit, Istituto Nazionale per la Ricerca sul Cancro, Largo Rosanna Benzi 10, 16132 Genova, Italy \\ Correspondence should be addressed to Andrea De Maria, de-maria@unige.it
}

Received 6 April 2011; Accepted 23 May 2011

Academic Editor: Lorenzo Moretta

Copyright ( $) 2011$ Francesco Marras et al. This is an open access article distributed under the Creative Commons Attribution License, which permits unrestricted use, distribution, and reproduction in any medium, provided the original work is properly cited.

\begin{abstract}
Natural Killer (NK) cells are endowed with cell-structure-sensing receptors providing inhibitory protection from self-destruction (inhibitory NK receptors, iNKRs, including killer inhibitory receptors and other molecules) and rapid triggering potential leading to functional cell activation by Toll-like receptors (TLRs), cytokine receptors, and activating NK cell receptors including natural cytotoxicity receptors (NCRs, i.e., NKp46, NKp46, and NKp44). NCR and NKG2D recognize ligands on infected cells which may be endogenous or may directly bind to some structures derived from invading pathogens. In this paper, we address the known direct or indirect interactions between activating receptors and pathogens and their expression during chronic HIV and HCV infections.
\end{abstract}

\section{Introduction}

Natural Killer (NK) cells represent a highly specialized lymphoid population initially identified by a potent cytolytic activity against tumor or virus infected cells. Different from T or B lymphocytes, they do not express clonally distributed receptors for antigen $[1,2]$, while their function is finely regulated by a balance of inhibitory and activating receptors. NK cell inhibitory receptors, recognizing mostly HLA class I molecules on "self" cells (notable exceptions to this concept are represented, among others, by Siglec7 and IRP60 recognizing non-HLA-related structures), turn NK cells "off" and represent the major failsafe device to prevent NK-mediated attack of normal HLA class $\mathrm{I}^{+}$autologous cells. On the other hand, the "on" signal is delivered when NK cells interact with target cells that lack MHC class I molecules and at the same time are triggered through activating molecules expressed by these cells (Figure 1). Activating stimuli may be delivered to NK cells through triggering via Toll-like receptors (TLRs) including TLR2, TLR3, TLR7/8, TLR9, interleukin receptors (IL-2, IL-12, IL-15, IL-18), and combinations thereof (e.g.,
IL-2 + IL-15, IL-2 + IL12, IL-12 + IL-18), or activatory receptors representing an array of different molecules expressed on their surface including natural cytotoxicity receptors (NCRs), NKG2D, NKG2C (a lectin-type triggering receptor which dimerizes with CD94), 2B4 (CD244), NKp80, DNAM1, NTB-A, and the receptor for IgFc (CD16) [3].

The receptors responsible for NK cell activation in the process of natural cytotoxicity are collectively termed natural cytoxicity receptors (NCRs): NKp46 [4, 5], NKp44 [6, 7], and NKp30 [8]. Their expression is mostly restricted to NK cells, and particularly in the case of NKp46, they represent the most accurate surface markers for human NK cell identification. Exceptions for NK cell identification have been documented. NKp44 may be detected on the surface of a minority of peripheral plasmacytoid dendritic cells [9] but on a relevant fraction of tissue-resident pDC [10] and NKp30 may be expressed by umbilical cord T-lymphocytes upon activation [11]. While NKp30 and NKp46 enable a precise identification of NK cells, regardless of whether these cells are resting or activated, NKp44 is selectively expressed only by activated NK cells $[6,7,12]$ and should be differentiated from 


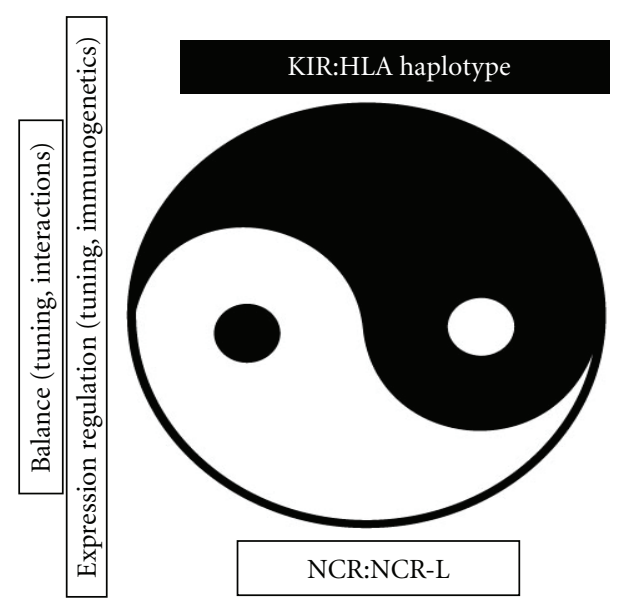

FIGURE 1: Diagram resuming the balance of activating and inhibitory NK cell receptor:ligand relationships.

NKp44 which is constitutively expressed on pDCs in tissues [10].

NCRs play a major role in NK-mediated killing of most tumor cell lines, as revealed by monoclonal antibody-mediated receptor-masking experiments $[3,12]$. Moreover, their surface density on NK cells correlates with the magnitude of cytolytic activity against NK-susceptible target cells [13]. The ligands recognized by NCRs are still incompletely molecularly defined and may have variable expression on different cells [14]. However, as revealed by cytolytic assays, they are expressed by cells belonging to different histotypes [2, 1517] and, in some cases, may be associated to neoplastic cells (e.g., B7-H6) [18] or to RNA viruses including influenza, dengue, or West Nile virus $[19,20]$. NKG2D is another major NK-cell-triggering receptor belonging to the NKG2 family (type II membrane proteins characterized by a lectinlike domain) [21-23]. Contrary to the NCR, NKG2D is not restricted to NK cells but may be also expressed by cytolytic T lymphocytes [24]. NKG2D is specific for stress-inducible polymorphic MHC-class-I-related chain (MIC), MIC-A and MIC-B or ULBP proteins [25], which may be expressed upon cell infection [26] or transformation [3].

Other triggering surface molecules expressed by NK cells are shared by other leucocyte types and appear to function primarily as coreceptors. They may function to amplify signaling by true receptors. Two such coreceptors, 2B4 [27] and NTB-A [28], appear to serve a dual and opposite function, depending of availability of downstream regulating elements on their signaling pathways.

A triggering surface molecule termed NKp80 has been identified by the generation of specific mAb (MA152 and LAP171) [29]. NKp80 is expressed by virtually all fresh NK cells derived from peripheral blood as well as by a minor T-cell subset characterized by the $\mathrm{CD}^{+}{ }^{+} \mathrm{CD} 56^{+}$surface phenotype [30] and binds to AICL on target cells [31]. NKp80 has so far no specific reactivity with pathogen-associated structures.

Another molecule behaving as triggering coreceptor in NK cells was described following attempts to identify the cellular ligands of triggering receptors [32]. DNAM-1 is a transmembrane protein involved in lymphocyte adhesion and signaling. In addition to NK cells, it is expressed also on T cells, monocytes, and a small subset of B lymphocytes. The role of DNAM-1 in NK-mediated killing varies with the different target cells analyzed thus far, suggesting differences in the expression of DNAM-1 ligands. Indeed, carcinomas and hematopoietic cell lines express PVR and Nectin-2, and their lysis involves DNAM-1. On the other hand, most EBVtransformed B cell lines analyzed do not express PVR nor Nectin-2, and their lysis does not involve DNAM-1 [32]. Thus, PVR and Nectin-2 represent the major (if not only) ligands of DNAM-1. As in the case of the other triggering receptors, the NK cell activation via DNAM-1 is controlled by HLA-class-I-specific inhibitory receptors. As a consequence, normal cells are usually protected from lysis.

Other triggering receptors including CD27, CRTAM, CD96, CD100(SEMA4D), PSGL1, and CD319 (SLAMF7) may be expressed by NK cells and have known ligands [3]. None of them has been so far reported to significantly interact with invading pathogens.

\section{NCR-Pathogen Interactions}

NK cells are a central component of innate immune response, comprising the first line of defense against a variety of tumors and microbial pathogens, including viruses, bacterial, fungal, and other intracellular parasites [33-36]. The lytic activity of NK cells is controlled by complex interactions of inhibitory and activating receptors with specialized signalling machinery, and at times, the possibility to directly detect pathogen-derived molecules, independent of Toll-like receptors $[2,37,38]$.

In addition to possible direct activation of NK cells via TLR by pathogen-derived molecular structures (PAMP, e.g., LPS, RNA, DNA), accumulating evidences over recent years have linked NCRs on NK cells with direct or indirect recognition of pathogen-associated structures. Given their role for sensing intracellular pathogen-infected cells, under particular conditions, these observations may bear relevant importance in the outcome of an immune response.

One should also consider that, even in the presence of weak inhibitory activity by KIR or other inhibitory receptors (e.g., KIR3DL2-HLA-C1 homozygosity), defects in NCR expression could lead to failure in recognizing pathogenassociated molecules acting as NCR ligands on infected cells, leading to variable degrees of derangement in NK cell function (Figure 2). The balance of KIR carriage:HLA class I expression and NKG2A/CD94:HLA-E or CD85j(LIR1/ ILT2):/HLA class I expression needs also to be considered in these cases. In other terms, although KIR:HLA carriage is known to influence NK cell activation [39], this pathway alone is not exhaustively representing overall NK cell function, for example in KIR-negative CD $56^{\text {bright }} \mathrm{NK}$ cells, in NK cell precursors (which are KIR-NKG2A+ and KIR-NKG2A-, resp.), or in CD56dull NK cells carrying KIR2DL3 on an HLA-C1 homozygous background but in addition express high levels of CD85J (LIR1/ILT2). 


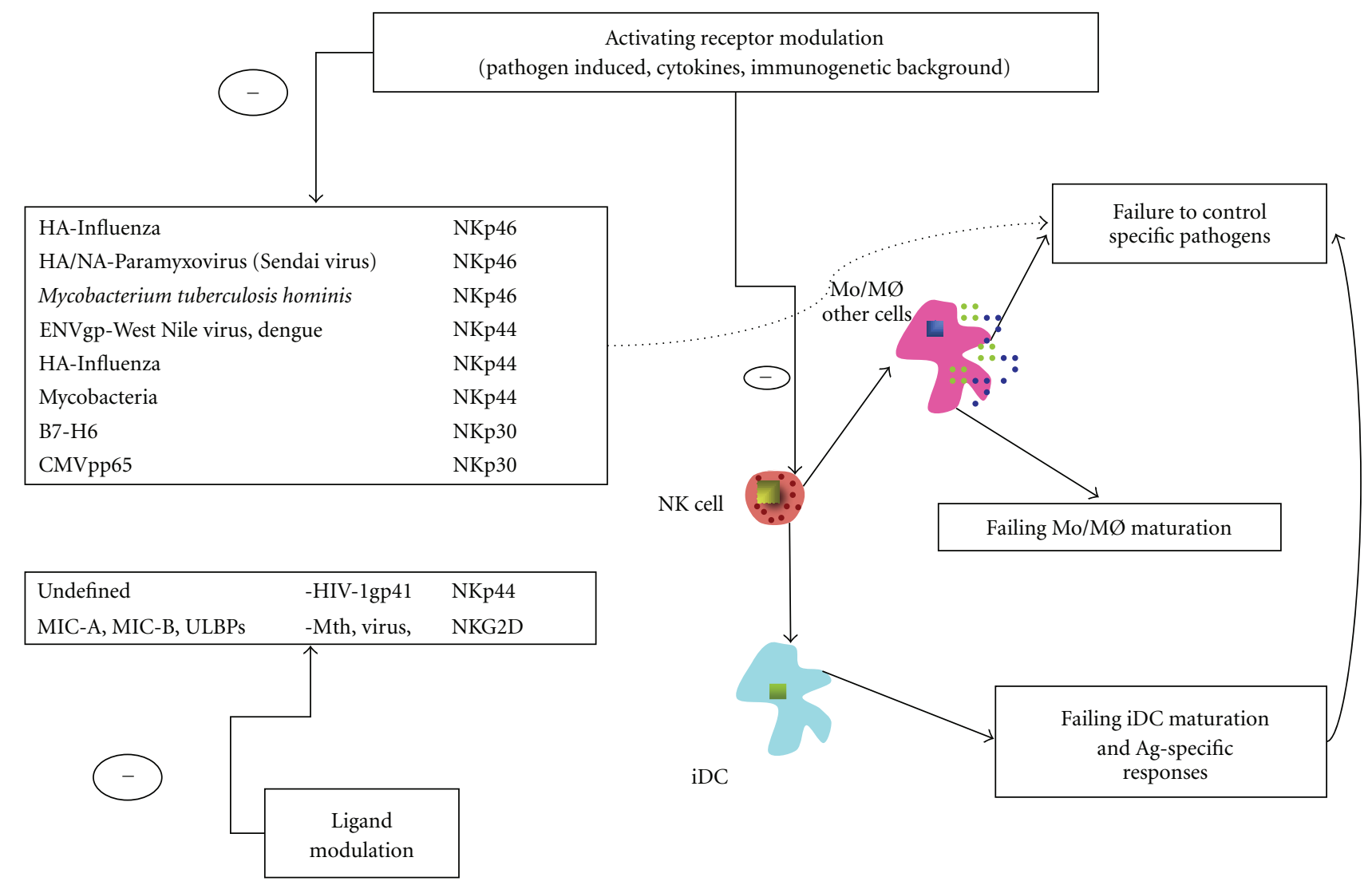

FIGURE 2: Diagram representing possible consequences of activating receptor modulation, alone, on the control of acute or chronic infections.

NCRs have been found to interact with infected cells through recognition of virus-encoded molecules. Sendai virus (SV) haemagglutinin-neuraminidase (HN) and Influenza virus (IV) haemagglutinin (HA) both recognize terminal $\mathrm{N}$-acetylneuraminic acid residues (sialic acids) attached to Gal. IV HA and SV HN, bind to sialic acid residues on NKp46 and NKp44 [19, 40], enabling NCR-mediated lysis of IV or SV-infected cells. Subsequent clinical observations corroborated these findings and showed that lethal IV infection in humans may be associated to absence of NKp46 on NK cells [41]. NKp46 is also involved in the detection and lysis of vaccinia-virus-infected cells, together with NKp30 and NKp44 [42] although viral ligands have not been characterized.

NKp44 has been reported to interact directly to cell-free mycobacteria or other bacteria [43] and to play a role in the recognition of virus-infected cells. In fact, following the original description of a putative interaction of NKp46 with influenza virus haemagglutinin and Sendai Virus hemagglutinin-neuraminidase [19], also NKp44 was found to interact with these proteins [40]. Direct interaction of NKp44 with virus-infected cells and with virus or virus-like particles, has been recently shown also with regard to the envelope protein of flavivirus (West Nile virus, dengue Virus) [20]. This leaves open the question of the potential relationship of HCV (another member of the Flaviviridae family) and NCR expression with regard to disease course. NKp44 has been involved in disease pathogenesis also with regard to HIV. HIV-1-infected CD4 ${ }^{+}$cells are reported to be targeted by NK cells via a NKp44L that is induced by an HIVgp41 peptide [44]. The presence of antibodies to this peptide in vivo would prevent $\mathrm{NK}$ cell recognition and disposal of infected cells. In HIV-infected patients, these antibodies are correlated with $\mathrm{CD} 4^{+}$cell numbers and NKp44L expression on $\mathrm{CD}^{+}$cells [45]. There has been so far no nearer characterization of this ligand, although it has been recently shown that the $3 \mathrm{~S}$ motif of gp 41 binds to $\mathrm{gClqR}$ (a receptor for the globular domain of $\mathrm{Clq}$ ) on $\mathrm{CD} 4^{+} \mathrm{T}$ cells and induces activation of a signalling cascade that leads to NKp44L expression also on uninfected $\mathrm{CD} 4^{+} \mathrm{T}$ cells [46]. Thus, NKp44 may be involved not only in protection from overwhelming infection by direct interaction with cells infected by viruses belonging to different virus families (Orthomyxo-, Paramyxo-, Flavi-, and Lentiviridae) but may also play a role in the pathogenesis of hyperactivation syndromes (as with influenza or dengue) or in immune depletion (e.g., HIV-1).

Several aspects remain be further clarified in this area, as direct NK-pathogen interaction via an activating receptor that is only expressed upon NK cell activation (NKp44), entails recognition only after full NK cell activation and not upon initial events following virus entry.

In line with what has been recently shown for NKp44, also NKG2D interacts with multiple cellular- or pathogenderived ligands that trigger NK cell cytotoxicity. NKG2D 
binds to the polymorphic MHC-class-I-related chain (MIC), MIC-A and MIC-B [47], and also binds to UL-16-binding proteins (ULBPs), which are MHC class-I-like molecules that are expressed by tumor cell lines. ULBPs enhance the capacity of NK cells to lyse tumors and to produce cytokines such as IFN $\gamma$ and TNF $\alpha[48,49]$ and are upregulated during heat shock or during infection with CMV [50,51].

Opposite to NKp44 and NKp46, there is little evidence for direct interaction of NKG2D with pathogen(s); however, its ligands may be either induced or downmodulated upon infection by several pathogens. In the case of rodent poxviruses such as ectromelia virus (mousepox virus), NKG2D ligands are upregulated on infected cells $[52,53]$, while it appears that human poxviruses such as vaccinia virus do not affect NKG2D expression in their host but rather affect NKp46, NKp44, and NKp30 through IFN- $\alpha$-mediated increased expression $[42,54]$. NKG2D appears to play a relevant role in killing HIV-1-infected $\mathrm{CD} 4^{+}$cells, as its ligands are still expressed in infected cells in vitro [26]. However, HIV Nef is specifically downmodulating ULBP2 [55], thus, preventing NKG2D-mediated cytotoxicity. Downregulation of NKG2D ligands MIC-A and MIC-B is also induced by the K5 immune evasion gene of KSHV (HHV-8). The same gene product also determines downregulation of the ligand of NKp80 (i.e., AICL), thus, simultaneously maiming both NK- and T-cell responses against HHV-8 at multiple levels [56]. Human CMV has developed a similar protective system against both NKG2D-expressing T cells and NK cells, as its UL16 protein binds to 3 or the main NHG2D ligands (i.e., MICB, ULBP1, ULBP2), thus, preventing them from being expressed by infected cells, thereby, avoiding cell killing by NKG2D-expressing NK cells [57]. A less extensive NKG2DLigand targeting (i.e., MICA alone) has been reported also for $\mathrm{HCV}$ via its NS3/4A serine protease [58]. Overall, there are still conflicting views in this area that could need additional attention to improve our understanding of the role played by NKG2D and NKG2D-interference during infection by intracellular pathogens comparing human and animal models. Since NKG2D is also expressed by a relevant proportion of $\mathrm{CD} 8^{+}$CTLs, additional aspects need do be addressed comprehensively to improve our understanding of the relative role of NK cells and CD8 CTLs in disease pathogenesis to help advise possible interventions.

There are few if any reports suggesting a direct interaction of other triggering coreceptors 2B4 (CD224) or DNAM- 1 or their ligands (i.e., CD48 and poliovirus receptor (CD155) and Nectin-2 (CD112) with viral products) [32, $59,60]$. One possible exception is represented by NKp80, as mentioned above, which is targeted by HHV-8 proteins which induce downmodulation of its ligand in infected cells.

\section{NCR Involvement in the Pathogenesis of NK Cell Dysfunction During HIV Infection}

The strongest evidence for a role of the immune system in controlling HIV-1 disease comes from a number of epidemiological studies demonstrating a strong influence of individual HLA class I alleles on determining the rate of HIV-1 disease progression [61]. Several subsets of cells belonging to the hematopoietic lineage express receptors that bind to HLA class I molecules, including $\mathrm{CD}^{+} \mathrm{T}$ cells, monocytes, dendritic cells, and NK cells.

A number of observations strongly suggest that $\mathrm{CD}^{+} \mathrm{T}$ cells play an important role in the containment of HIV infection. For example, exposed-uninfected seronegative subjects lack specific humoral responses to HIV-1 but have detectable $\mathrm{CD} 8^{+} \mathrm{CTL}$ responses and mucosal IgA immunity [62-65]. However, HIV-1-specific $\mathrm{CD}^{+}{ }^{+}$T-cell immunity alone is not sufficient to explain the large heterogeneity observed in the clinical manifestation of HIV-1 disease. More recent advances in the understanding of the immune response to viral infections support the involvement of additional components of the innate immune system in the control of HIV-1 disease and might help to identify the mechanism underlying protective immunity in HIV-1 infection.

HIV-1 infection is associated with significant changes in NK cell subset distributions and function in the peripheral circulation which were detected already at the beginning of the epidemic and were subsequently systematically evaluated and reviewed [66-68]. Several reports have shown a dramatic reduction in the proportion of $\mathrm{CD}^{-} \mathrm{CD}^{-} 6^{+}$ NK cells $[69,70]$, and particularly $\mathrm{CD} 56^{\text {bright }} \mathrm{CD} 16^{+/-}[71]$. This reduction appears to be partially attributable to the emergence of a novel subset of NK cells that is rare in healthy individuals, $\mathrm{CD}^{-} \mathrm{CD}^{-} 6^{-} \mathrm{CD} 16^{+} \mathrm{NK}$ cells $[68,72]$. Earlier work showed that NK cells in HIV-viremic patients displayed a functionally relevant and dramatic reduction in NCRs [67], which is accompanied by relevant activation, as determined by HLA-DR and CD69 expression [73]. A consistent fraction of these cells fall also in the subsequently identified $\mathrm{CD}^{-} 6^{-}$ $\mathrm{CD} 16^{+} \mathrm{CD}^{-}$"exausted" NK cell subset [72]. Since this subset becomes prominent in individuals with active viral replication at the expense of the two other subsets of cells (i.e., CD56 ${ }^{\text {dim }}$ and CD56 ${ }^{\text {bright }} \mathrm{NK}$ cells), it needs to be accounted for to evaluate the overall number of NK cells in patients with HIV-1 infection. NK cells with defective NCR expression in HIV patients, among these also $\mathrm{CD}^{-} \mathrm{CD}^{-} 6^{+} \mathrm{CD} 16^{+}$ NK cells, have strongly reduced NK cell effector functions, including killing, cytokine secretion, and antibodydependent cellular cytotoxicity (ADCC), and exhibit aberrant DC-editing activity $[67,73,74]$. DC-editing by NK cells may take place whenever DCs and NK cells interact in secondary lymphoid organs. DCs that do not express functionally relevant HLA class I molecule densities are subject to NK cell killing (e.g., iDCs and incompletely mature DCs). NK cells in turn induce DC maturation through IFN- $\gamma$ and TNF$\alpha$ production [75-79]. Since incompletely mature DCs produce higher levels of IL-10 and lower quantities of IL-12 and show less efficient antigen presentation [80], the efficiency of NK-DC interactions "crosstalk" including cytokine production and NKp30/DNAM-1 expression may variably shape subsequent downstream T-cell and B-cell adaptive responses.

In addition to changes in NK subpopulations associated with HIV infection, there are also marked changes in NK surface receptor expression that are related to loss of function. With HIV viremia, there is an overall decrease in surface receptor density of NKp46 and NKp30 found on freshly 
drawn NK cells and dysfunction in NKp44 de novo expression upon stimulation in vitro resulting in what has been termed an $\mathrm{NCR}^{\text {dull }}$ phenotype [67]. In the $\mathrm{CD} 56^{\mathrm{dim}}$ subset, the proportion of cells expressing NKp46 and NKp30 and their cytolytic activity decrease with disease progression [81]. Moreover, a concomitant increase of KIR density is also observed not only on $\mathrm{CD}^{+} \mathrm{CTL}$, but also on NK cells, thus, setting the basis for an increased inhibitory potential of cytolytic cells [82-84]. The downmodulation of activating receptors and upregulation of KIRs result in measurable functional defects in NK-cell-mediated cytotoxicity regardless of the NK cell subpopulation.

The overall cause of NK cell dysfunction during HIV infection is poorly understood. The induction of NKp44L by HIVgp41 on infected and uninfected $\mathrm{CD}^{+}$cells and the as yet poorly characterized downmodulation of activatory receptor and coreceptor ligands are likely to be involved [26, 46]. Despite isolated reports on the possibility of infection of CD4 ${ }^{-}$expressing or activated NK cells $[85,86]$, peripheral NK cells are not infected by HIV [72]. This phenomenon may be rather attributed to cells different from NK cells [87] and is currently not regarded as a major mechanism leading to NK cell dysfunction.

Antiretroviral treatment (ART) does not significantly affect NK cell function recovery, as IFN $\gamma$ production [88], and NCR expression may be persistently impaired even after successful ART and virus control in patients with $\mathrm{CD} 4^{+}$ cell numbers $>500 / \mu \mathrm{L}$ [89]. Variable NCR expression during ART, thus, appears to contribute to clinical disease course upon treatment interruption. Thus, differences in innate immune balance during ART may be associated to differential control of HIV. Their understanding could explain clinical differences in individual patients that are not reflected by $\mathrm{CD} 4^{+}$cell counts alone.

Overall, therefore, although KIR:HLA carriage may significantly affect the course of HIV infection [61, 90, 91], direct or indirect disruption of NCR/NCR-ligand expression by HIV represents a significant event that needs to be considered in parallel when evaluating NK cell activating/inhibiting balance. Disruption of NK-DC interaction and DC editing by NK cells via NKp30 [76, 78, 79] and DNAM-1 [77] is likely to lead to inefficient selection of DC maturation with inefficient downstream antigen-specific $\mathrm{T}$ - and B-cell responses [74, 92].

\section{NCRs in Nonhuman Primates and HIV/SIV Infection}

Studies describing NKp46, NKp30, NKG2D, and NKp80 in animal models of HIV infection revealed that NCR expression in SHIV-infected macaques does not reflect the downmodulation observed in HIV-infected humans [93, 94]. Importantly, defective transcription of NKp44 has been observed in macaques and this explains why no surface expression of this NCR is detected in macaques (Macaca mulatta and Macaca fascicularis) [93-95], thus, raising questions on the interpretation of some experiments in this animal model with regard to NK cell regulation.
On the contrary, although NKp44 transcription and induction appears to be differently regulated in chimpanzees [95] compared to humans, this primate species has a full set of NCRs [96]. A relevant difference with Homo sapiens relies in NKp30 expression and regulation and may impact on the relative resistance to progressive HIV infection in this species. Indeed, in both uninfected and HIV-infected chimpanzees $\mathrm{NKp} 30$ is not- or poorly expressed but is de novo expressed at levels comparable to human NK cells upon cell activation [96]. Thus, even during HIV replication, NK:DC crosstalk via NKp30 is likely to be dampened and may explain the low level of immune activation even in the presence of active replication of a virus that in humans thrives on cell activation.

\section{NCRs and NK Cell Function in HCV Infection}

NK cells have been implicated in all stages of HCV infection in both genetic and functional studies. This role may be either direct, by targeting hepatocytes, or indirect by influencing other key immunocytes such as DCs or T cells. NK cells comprise $5-20 \%$ of peripheral blood mononuclear cells but make up a substantially greater proportion (30$50 \%)$ of lymphocytes in the liver [97]. Intrahepatic NK cells in murine models may behave differently (i.e., hyporesponsiveness) compared to NK cells in other districts, due to a presumed "tolerogenic" environment in the liver. They are less cytotoxic and have an altered cytokine profile producing lower level of IFN $\gamma$ and greater levels of Immunomodulatory cytokines, such as IL-10, compared to peripheral blood and splenic NK cells [98].

Human peripheral blood NK cells are involved in the acute phase of HCV infection, with an increase in CD $56^{\text {bright }}$ NK cells and associated reduction in CD56 $6^{\mathrm{dim}}$ subset [99]. A decline in the CD56 $6^{\text {bright }}$ population is observed in patients spontaneously clearing the virus and reaching levels comparable to healthy control individuals within 1-3 months. This return to baseline is not observed in those that proceed to chronic HCV infection. In the acute phase of infection, expression of NKG2D is increased with augmented IFN $\gamma$ production and cytotoxicity [99]. In addition, peak NK cell activation and degranulation precedes or coincides with peak T-cell responses, and a correlation was observed between NK cell degranulation and the magnitude of HCV-specific T-cell responses [100]. Thus, recent evidences agree with a direct involvement of NK cell responses during acute HCV infection favoring induction and priming of downstream T-cell responses leading to virus clearance.

During chronic HCV infection, peripheral blood NK cell frequency (both absolute number and percentage of total lymphocytes) is reduced in chronic HCV compared with healthy individuals [101-104]. NK cell frequency increases following successful antiviral therapy [105]. A reduction in peripheral blood NK cell frequency in patients with chronic HCV as compared to spontaneous resolvers has also been noted $[106,107]$. These observations may be at least in part explained by a significant reduction of IL-15 (a pivotal cytokine for NK cell development, proliferation, 
and function) concentrations/levels which is observed in HCV patients as compared to healthy controls [103].

Several studies in chronic HCV patients have documented a relative increase in circulating CD56 $6^{\text {bright }}$ (but not CD56 ${ }^{\text {dim }}$ NK cells) compared to healthy individuals and spontaneous resolvers $[102,106,108]$. Bonorino et al. demonstrated that the relative proportions of CD56 ${ }^{\mathrm{dim}}$ and CD56 ${ }^{\text {bright }}$ NK cells in the liver are altered in chronic $\mathrm{HCV}$ observing that $80.5 \%$ of intrahepatic NK cells were CD $56^{\mathrm{dim}}$ as compared to $94 \%$ in peripheral blood, and $19.5 \%$ intrahepatic NK cells were CD56 ${ }^{\text {bright }}$ as compared to $6.0 \%$ in peripheral blood [102]. This implies that the decreased frequency of $\mathrm{CD} 56^{\mathrm{dim}}$ in the periphery is not related to their sequestration in the liver, although there are no nearer data on the relative proportion of $\mathrm{CD} 56^{\mathrm{dim}}$ and $\mathrm{CD} 56^{\text {bright }} \mathrm{NK}$ cells in the healthy liver. Changes in NK cell phenotype may not necessarily reflect changes in subset distribution. CD56 ${ }^{\text {bright }}$ NK cells are KIR-negative and NKG2A-positive, and one of the most consistent findings so far has been an increase in NKG2A expression in chronic HCV infection [108-110]. This occurs on both intrahepatic and peripheral blood NK populations [102] and also involves CD56 ${ }^{\mathrm{dim}} \mathrm{NK}$ cells.

Contrary to HIV infection, there has been so far less consensus on NCR expression during chronic HCV infection. Increased proportions and density of NCRs, including NKG2C, NKp44, NKp30, and NKp46 have been reported $[108,111,112]$. Initial reports of decreased expression of NKp46 [113] have not been subsequently confirmed. Similarly, there is conflicting evidence with respect to NKG2D expression which has been reported as being upregulated, downregulated, and also unchanged during chronic HCV infection $[108,109,112]$. However, these apparently opposite findings could be reconciled by different treatment responses in different cohorts [114].

Thus, the interaction of HCV with NCRs on NK cells is less direct compared to HIV, as direct virus challenge does not impair NK cell function [115], and may be mediated by other as yet poorly understood viral factors [116] or by possible immunogenetic traits that would parallel those observed for the other side of the (NK cell) coin (i.e., KIR:HLA interactions) [117].

\section{NCRs during Mycobacterium tuberculosis hominis Infection}

Different sets of observations support the notion that NK cells may be involved in the control of mycobacterial infections, with particular emphasis on Mycobacterium tuberculosis hominis (Mth). First, NK cells from healthy donors (HDs) can directly respond to products via TLR-2 [118]. Moreover, NK cells respond to mycobacterium-infected $\mathrm{mDC}$ [75]. Direct interaction between NCRs and Mth has been suggested (see above). A direct link between NK cells and Mth-infected cells has been shown to be mediated by the interaction of NKp46- and NKG2D-activating receptors on NK cells with infected monocytes [119]. Evidences indicating a relevant role for NK-mediated control of Mth replication are provided by experiments using cells from HDs showing their promotion of intracellular killing of mycobacteria [120, 121] and by their ability to lyse monocytes infected with Mth, Mycobacterium tuberculosis bovis Bacille Calmette-Guerin or Mycobacterium avium intracellulare [122]. In addition, the recent description of reduced $\mathrm{mDC}$ and $\mathrm{pDC}$ numbers and function in patients with TB [123] suggests that this, in turn, may impair NK cell function (e.g., through impaired production of cytokines promoting NK cell activation such as IL-12 and IL-15) contributing to reduce lysis of Mthinfected macrophages which are, in turn, subjected to the activation of antiapoptotic pathways by Mth $[119,124]$.

NK cells have been shown to play a fundamental role in the maintenance of efficient Ag-specific CD $8^{+}$CTL responses in healthy humans with latent Mth infection [125]. In line with these observations, patients with recent onset of pulmonary TB have low-level expression of both NKp46 and NKp30 [126], suggesting that NK-cell-driven downstream adaptive responses may also be defective. In addition, patients successfully recovering from pulmonary $\mathrm{TB}$ with standard treatment (rifampicin, isoniazid, ethambutol, and pyrazinamide for 2 months followed by 24 months of rifampicin and isoniazid) do recover IFN $\gamma$ production by NK cells but fail to recover NKp30 and NKp46 expression [126] and in some cases do so even after 3-5 years from end of treatment (A. De Maria, personal observation). These observations could be explained by the hypothetical scenario of immunogenetically determined regulation on NCR expression that may influence different interindividual susceptibility to latent Mth reactivation with onset of disease.

\section{Concluding Remarks}

So far, the role of NCRs in the interaction of pathogens with NK cells has been largely considered ancillary to cytokine- or TLR-mediated activation and to KIR:HLA modulation of NK cell function. Over the last 10 years accumulating evidences point to the possibility of viruses to directly interact with NCRs to tamper with their expression and to interfere with NCR ligand expression on target cells, thus, indirectly reducing NK cell function irrespective of the KIR:HLA haplotype (Figure 2).

Improving our knowledge of the mechanism(s) and of the regulation of NCR expression and function and integrating this information with inhibitory receptor expression and function will improve the prospective management of patients with potentially lethal or invalidating acute infections including influenza, dengue, or HCV and optimize treatment strategies of chronic invalidating persistent infections such as HIV, TB, and HCV.

\section{References}

[1] G. Trinchieri, "Biology of natural killer cells," Advances in Immunology, vol. 47, pp. 187-376, 1989.

[2] L. Moretta, R. Biassoni, C. Bottino, M. C. Mingari, and A. Moretta, "Human NK-cell receptors," Immunology Today, vol. 21, no. 9, pp. 420-422, 2000. 
[3] E. Vivier, D. H. Raulet, and A. Moretta, "Innate or adaptive immunity? The example of natural killer cells," Science, vol. 331, no. 6013, pp. 44-49, 2011.

[4] S. Sivori, M. Vitale, L. Morelli et al., "p46, a novel natural killer cell-specific surface molecule that mediates cell activation," Journal of Experimental Medicine, vol. 186, no. 7, pp. 1129-1136, 1997.

[5] A. Pessino, S. Sivori, C. Bottino et al., "Molecular cloning of NKp46: A novel member of the immunoglobulin superfamily involved in triggering of natural cytotoxicity," Journal of Experimental Medicine, vol. 188, no. 5, pp. 953-960, 1998.

[6] M. Vitale, C. Bottino, S. Sivori et al., "NKp44, a novel triggering surface molecule specifically expressed by activated natural killer cells, is involved in non-major histocompatibility complex-restricted tumor cell lysis," Journal of Experimental Medicine, vol. 187, no. 12, pp. 2065-2072, 1998.

[7] C. Cantoni, C. Bottino, M. Vitale et al., "NKp44, a triggering receptor involved in tumor cell lysis by activated human natural killer cells, is a novel member of the immunoglobulin superfamily," Journal of Experimental Medicine, vol. 189, no. 5, pp. 787-795, 1999.

[8] D. Pende, S. Parolini, A. Pessino et al., "Identification and molecular characterization of NKp30, a novel triggering receptor involved in natural cytotoxicity mediated by human natural killer cells," Journal of Experimental Medicine, vol. 190, no. 10, pp. 1505-1516, 1999.

[9] A. Fuchs, M. Cella, T. Kondo, and M. Colonna, "Paradoxic inhibition of human natural interferon-producing cells by the activating receptor NKp44," Blood, vol. 106, no. 6, pp. 2076-2082, 2005.

[10] I. Bonaccorsi, C. Cantoni, P. Carrega et al., "The immune inhibitory receptor LAIR-1 is highly expressed by plasmacytoid dendritic cells and acts complementary with NKp44 to control IFN $\alpha$ production," PLoS ONE, vol. 5, no. 11, Article ID e15080, 2010.

[11] Q. Tang, B. Grzywacz, H. Wang et al., "Umbilical cord blood $\mathrm{T}$ cells express multiple natural cytotoxicity receptors after IL-15 stimulation, but only NKp30 is functional," Journal of Immunology, vol. 181, no. 7, pp. 4507-4515, 2008.

[12] R. Biassoni, C. Cantoni, D. Pende et al., "Human natural killer cell receptors and co-receptors," Immunological Reviews, vol. 181, pp. 203-214, 2001.

[13] S. Sivori, S. Parolini, M. Falco et al., "2B4 functions as a coreceptor in human NK cell activation," European Journal of Immunology, vol. 30, no. 3, pp. 787-793, 2000.

[14] A. Byrd, S. C. Hoffman, M. Jarahian, F. Momburg, and C. Watzl, "Expression analysis of the ligands for the natural killer cell receptors NKp30 and NKp44," PLoS ONE, vol. 2, no. 12, Article ID e1339, 2007.

[15] S. Sivori, S. Parolini, E. Marcenaro et al., "Involvement of natural cytotoxicity receptors in human natural killer cellmediated lysis of neuroblastoma and glioblastoma cell lines," Journal of Neuroimmunology, vol. 107, no. 2, pp. 220-225, 2000.

[16] R. T. Costello, S. Sivori, E. Marcenaro et al., "Defective expression and function of natural killer cell-triggering receptors in patients with acute myeloid leukemia," Blood, vol. 99, no. 10, pp. 3661-3667, 2002.

[17] D. Pende, P. Rivera, S. Marcenaro et al., "Major histocompatibility complex class I-related chain A and UL16-binding protein expression on tumor cell lines of different histotypes," Cancer Research, vol. 62, no. 21, pp. 6178-6186, 2002.

[18] C. S. Brandt, M. Baratin, E. C. Yi et al., "The B7 family member B7-H6 is a tumor cell ligand for the activating natural killer cell receptor NKp30 in humans," Journal of Experimental Medicine, vol. 206, no. 7, pp. 1495-1503, 2009.

[19] O. Mandelboim, N. Lieberman, M. Lev et al., "Recognition of haemagglutinins on virus-infected cells by NKp46 activates lysis by human NK cells," Nature, vol. 409, no. 6823, pp. 1055-1060, 2001.

[20] O. Hershkovitz, B. Rosental, L. A. Rosenberg et al., "NKp44 receptor mediates interaction of the envelope glycoproteins from the West Nile and dengue viruses with NK cells," Journal of Immunology, vol. 183, no. 4, pp. 2610-2621, 2009.

[21] J. Wu, Y. Song, A. B. H. Bakker et al., "An activating immunoreceptor complex formed by NKG2D and DAP10," Science, vol. 285, no. 5428, pp. 730-732, 1999.

[22] A. Cerwenka and L. L. Lanier, "Ligands for natural killer cell receptors: redundancy or specificity," Immunological Reviews, vol. 181, pp. 158-169, 2001.

[23] A. Diefenbach, E. R. Jensen, A. M. Jamieson, and D. H. Raulet, "Rae1 and H60 ligands of the NKG2D receptor stimulate tumour immunity," Nature, vol. 413, no. 6852, pp. 165$171,2001$.

[24] V. Groh, R. Rhinehart, J. Randolph-Habecker, M. Topp, S. Riddell, and T. Spies, "Costimulation of CD8alphabeta T cell by NKG2D via engagement by MIC induced on virusinfected cells," Nature Immunology, vol. 2, no. 3, pp. 255-260, 2001.

[25] C. L. Sutherland, N. J. Chalupny, and D. Cosman, "The UL16-binding proteins, a novel family of MHC class I-related ligands for NKG2D, activate natural killer cell functions," Immunological Reviews, vol. 181, pp. 185-192, 2001.

[26] J. Ward, M. Bonaparte, J. Sacks et al., "HIV modulates the expression of ligands important in triggering natural killer cell cytotoxic responses on infected primary T-cell blasts," Blood, vol. 110, no. 4, pp. 1207-1214, 2007.

[27] S. Parolini, C. Bottino, M. Falco et al., "X-linked lymphoproliferative disease," Journal of Experimental Medicine, vol. 192, no. 3, pp. 337-346, 2000.

[28] C. Bottino, M. Falco, S. Parolini et al., "NTB-A [correction of GNTB-A], a novel SH2D1A-associated surface molecule contributing to the inability of natural killer cells to kill EpsteinBarr virus-infected B cells in X-linked lymphoproliferative disease," Journal of Experimental Medicine, vol. 194, no. 3, pp. 235-246, 2001.

[29] M. Vitale, M. Falco, R. Castriconi et al., "Identification of NKp80, a novel triggering molecule expressed by human NK cells," European Journal of Immunology, vol. 31, no. 1, pp. 233-242, 2001.

[30] S. Kuttruff, S. Koch, A. Kelp, G. Pawelec, H. G. Rammensee, and A. Steinle, "NKp80 defines and stimulates a reactive subset of CD8 T cells," Blood, vol. 113, no. 2, pp. 358-369, 2009.

[31] S. Welte, S. Kuttruff, I. Waldhauer, and A. Steinle, "Mutual activation of natural killer cells and monocytes mediated by NKp80-AICL interaction," Nature Immunology, vol. 7, no. 12, pp. 1334-1342, 2006.

[32] C. Bottino, R. Castriconi, D. Pende et al., "Identification of PVR (CD155) and Nectin-2 (CD112) as cell surface ligands for the human DNAM-1 (CD226) activating molecule," Journal of Experimental Medicine, vol. 198, no. 4, pp. 557-567, 2003.

[33] J. S. Orange, B. Wang, C. Terhorst, and C. A. Biron, "Requirement for natural killer cell-produced interferon $\gamma$ in defense against murine cytomegalovirus infection and enhancement of this defense pathway by interleukin 12 administration," 
Journal of Experimental Medicine, vol. 182, no. 4, pp. 10451056, 1995.

[34] C. H. Tay, E. Szomolanyi-Tsuda, and R. M. Welsh, "Control of infections by NK cells," Current Topics in Microbiology and Immunology, vol. 230, pp. 193-220, 1998.

[35] D. L. Doolan and S. L. Hoffman, "IL-12 and NK cells are required for antigen-specific adaptive immunity against malaria initiated by CD8 $+\mathrm{T}$ cells in the plasmodium yoelii model," Journal of Immunology, vol. 163, no. 2, pp. 884-892, 1999.

[36] L. L. Ma, G. G. Neely, S. Epelman, A. M. Krensky, and C. H. Mody, "NK cells use perforin rather than granulysin for anticryptococcal activity," Journal of Immunology, vol. 173, no. 5, pp. 3357-3365, 2004.

[37] H. Arase, E. S. Mocarski, A. E. Campbell, A. B. Hill, and L. L. Lanier, "Direct recognition of cytomegalovirus by activating and inhibitory NK cell receptors," Science, vol. 296, no. 5571, pp. 1323-1326, 2002.

[38] L. L. Lanier, "On guard-Activating NK cell receptors," Nature Immunology, vol. 2, no. 1, pp. 23-27, 2001.

[39] S. I. Khakoo, C. L. Thio, M. P. Martin et al., "HLA and NK cell inhibitory receptor genes in resolving hepatitis $\mathrm{C}$ virus infection," Science, vol. 305, no. 5685, pp. 872-874, 2004.

[40] T. I. Arnon, M. Lev, G. Katz, Y. Chernobrov, A. Porgador, and O. Mandelboim, "Recognition of viral hemagglutinins by NKp44 but not by NKp30," European Journal of Immunology, vol. 31, no. 9, pp. 2680-2689, 2001.

[41] R. Gazit, R. Gruda, M. Elboim et al., "Lethal influenza infection in the absence of the natural killer cell receptor gene Ncr1," Nature Immunology, vol. 7, no. 5, pp. 517-523, 2006.

[42] S. E. Chisholm and H. T. Reyburn, "Recognition of vaccinia virus-infected cells by human natural killer cells depends on natural cytotoxicity receptors," Journal of Virology, vol. 80, no. 5, pp. 2225-2233, 2006.

[43] S. Esin, G. Batoni, C. Counoupas et al., "Direct binding of human NK cell natural cytotoxicity receptor NKp44 to the surfaces of mycobacteria and other bacteria," Infection and Immunity, vol. 76, no. 4, pp. 1719-1727, 2008.

[44] V. Vieillard, J. L. Strominger, and P. Debré, "NK cytotoxicity against CD4+ T cells during HIV-1 infection: a gp41 peptide induces the expression of an NKp44 ligand," Proceedings of the National Academy of Sciences of the United States of America, vol. 102, no. 31, pp. 10981-10986, 2005.

[45] V. Vieillard, D. Costagliola, A. Simon, and P. Debré, "Study FAàLTA. Specific adaptive humoral response against a gp41 motif inhibits CD4 T-cell sensitivity to NK lysis during HIV1 infection," AIDS, vol. 20, no. 14, pp. 1795-1804, 2006.

[46] H. Fausther-Bovendo, V. Vieillard, S. Sagan, G. Bismuth, and P. Debré, "Hiv gp41 engages gclqr on cd4 $+\mathrm{t}$ cells to induce the expression of an nk ligand through the pip3/h2o2 pathway," PLoS Pathogens, vol. 6, no. 7, Article ID e1000975, pp. 1-14, 2010.

[47] S. Bauer, V. Groh, J. Wu et al., "Activation of NK cells and T cells by NKG2D, a receptor for stress- inducible MICA," Science, vol. 285, no. 5428, pp. 727-729, 1999.

[48] M. Kubin, L. Cassiano, J. Chalupny et al., "ULBP1, 2, 3: novel MHC class I-related molecules that bind to human cytomegalovirus glycoprotein UL16, activate NK cells," European Journal of Immunology, vol. 31, no. 5, pp. 1428-1437, 2001.

[49] D. Cosman, J. Müllberg, C. L. Sutherland et al., "ULBPs, novel MHC class I-related molecules, bind to CMV glycoprotein UL16 and stimulate NK cytotoxicity through the NKG2D receptor," Immunity, vol. 14, no. 2, pp. 123-133, 2001.

[50] V. Groh, S. Bahram, S. Bauer, A. Herman, M. Beauchamp, and T. Spies, "Cell stress-regulated human major histocompatibility complex class I gene expressed in gastrointestinal epithelium," Proceedings of the National Academy of Sciences of the United States of America, vol. 93, no. 22, pp. 1244512450, 1996.

[51] V. Groh, R. Rhinehart, H. Secrist, S. Bauer, K. H. Grabstein, and T. Spies, "Broad tumor-associated expression and recognition by tumor-derived gamma delta T cells of MICA and MICB," Proceedings of the National Academy of Sciences of the United States of America, vol. 96, no. 12, pp. 6879-6884, 1999.

[52] M. Fang, L. L. Lanier, and L. J. Sigal, "A role for NKG2D in NK cell-mediated resistance to poxvirus disease," PLoS Pathogens, vol. 4, no. 2, Article ID e30, 2008.

[53] J. A. Campbell, D. S. Trossman, W. M. Yokoyama, and L. N. Carayannopoulos, "Zoonotic orthopoxviruses encode a high-affinity antagonist of NKG2D," Journal of Experimental Medicine, vol. 204, no. 6, pp. 1311-1317, 2007.

[54] P. Costa, S. Sivori, F. Bozzano et al., "IFN- $\alpha$-mediated increase in cytolytic activity of maturing NK cell upon exposure to HSV-infected myelomonocytes," European Journal of Immunology, vol. 39, no. 1, pp. 147-158, 2009.

[55] C. Cerboni, F. Neri, N. Casartelli et al., "Human immunodeficiency virus 1 Nef protein downmodulates the ligands of the activating receptor NKG2D and inhibits natural killer cellmediated cytotoxicity," Journal of General Virology, vol. 88, no. 1, pp. 242-250, 2007.

[56] M. Thomas, J. M. Boname, S. Field et al., "Down-regulation of NKG2D and NKp80 ligands by Kaposi's sarcomaassociated herpesvirus K5 protects against NK cell cytotoxicity," Proceedings of the National Academy of Sciences of the United States of America, vol. 105, no. 5, pp. 1656-1661, 2008.

[57] C. Dunn, N. J. Chalupny, C. L. Sutherland et al., "Human cytomegalovirus glycoprotein UL16 causes intracellular sequestration of NKG2D ligands, protecting against natural killer cell cytotoxicity," Journal of Experimental Medicine, vol. 197, no. 11, pp. 1427-1439, 2003.

[58] C. Wen, X. He, H. Ma et al., "Hepatitis C virus infection downregulates the ligands of the activating receptor NKG2D," Cellular \& Molecular Immunology, vol. 5, no. 6, pp. 475-478, 2008.

[59] M. Z. Kubin, D. L. Parshley, W. Din et al., "Molecular cloning and biological characterization of NK cell activationinducing ligand, a counterstructure for CD48," European Journal of Immunology, vol. 29, no. 11, pp. 3466-3477, 1999.

[60] H. Nakajima, M. Cella, H. Langen, A. Friedlein, and M. Colonna, "Activating interactions in human NK cell recognition: the role of 2B4-CD48," European Journal of Immunology, vol. 29, no. 5, pp. 1676-1683, 1999.

[61] M. Carrington and S. J. O’Brien, "The Influence of HLA genotype on AIDS," Annual Review of Medicine, vol. 54, pp. 535-551, 2003.

[62] M. L. Newell, D. Dunn, A. De Maria et al., "Detection of virus in vertically exposed HIV-antibody-negative children," The Lancet, vol. 347, no. 8996, pp. 213-215, 1996.

[63] S. L. Rowland-Jones, D. F. Nixon, M. C. Aldhous et al., "HIV-specific cytotoxic T-cell activity in an HIV-exposed but uninfected infants," The Lancet, vol. 341, no. 8849, pp. 860861, 1993.

[64] A. De Maria, C. Cirillo, and L. Moretta, "Occurrence of human immunodeficiency virus type 1 (HIV-1)-specific cytolytic $\mathrm{T}$ cell activity in apparently uninfected children born to 
HIV-1- infected mothers," Journal of Infectious Diseases, vol. 170, no. 5, pp. 1296-1299, 1994.

[65] R. Kaul, F. Plummer, M. Clerici, M. Bomsel, L. Lopalco, and K. Broliden, "Mucosal IgA in exposed, uninfected subjects: evidence for a role in protection against HIV infection," AIDS, vol. 15, no. 3, pp. 431-432, 2001.

[66] G. Poli, M. Introna, and F. Zanaboni, "Natural killer cells in intravenous drug abusers with lymphadenopathy syndrome," Clinical and Experimental Immunology, vol. 62, no. 1, pp. 128-135, 1985.

[67] A. De Maria, M. Fogli, P. Costa et al., "The impaired NK cell cytolytic function in viremic HIV-1 infection is associated with a reduced surface expression of natural cytotoxicity receptors (NKp46, NKp30 and NKp44)," European Journal of Immunology, vol. 33, no. 9, pp. 2410-2418, 2003.

[68] G. Alter, N. Teigen, B. T. Davis et al., "Sequential deregulation of NK cell subset distribution and function starting in acute HIV-1 infection," Blood, vol. 106, no. 10, pp. 3366-3369, 2005.

[69] B. G. Brenner, A. Dascal, R. G. Margolese, and M. A. Wainberg, "Natural killer cell function in patients with acquired immunodeficiency syndrome and related diseases," Journal of Leukocyte Biology, vol. 46, no. 1, pp. 75-83, 1989.

[70] M. B. Lucia, N. Froio, E. Tacconelli et al., "CD16+CD56+ CD8+ natural killer (NK) cells are decreased during HIV infection," European Journal of Histochemistry, vol. 41, supplement 2, pp. 197-198, 1997.

[71] M. R. Goodier, N. Imami, G. Moyle, B. Gazzard, and F. Gotch, "Loss of the CD56hiCD16- NK cell subset and NK cell interferon-gamma production during antiretroviral therapy for HIV-1: partial recovery by human growth hormone," Clinical and Experimental Immunology, vol. 134, no. 3, pp. 470-476, 2003.

[72] D. Mavilio, G. Lombardo, J. Benjamin et al., "Characterization of CD56-/CD16+ natural killer (NK) cells: A highly dysfunctional NK subset expanded in HIV-infected viremic individuals," Proceedings of the National Academy of Sciences of the United States of America, vol. 102, no. 8, pp. 2886-2891, 2005.

[73] M. Fogli, P. Costa, G. Murdaca et al., "Significant NK cell activation associated with decreased cytolytic function in peripheral blood of HIV-1-infected patients," European Journal of Immunology, vol. 34, no. 8, pp. 2313-2321, 2004.

[74] D. Mavilio, G. Lombardo, A. Kinter et al., "Characterization of the defective interaction between a subset of natural killer cells and dendritic cells in HIV-1 infection," Journal of Experimental Medicine, vol. 203, no. 10, pp. 2339-2350, 2006.

[75] G. Ferlazzo, B. Morandi, A. D’Agostino et al., "The interaction between NK cells and dendritic cells in bacterial infections results in rapid induction of NK cell activation and in the lysis of uninfected dendritic cells," European Journal of Immunology, vol. 33, no. 2, pp. 306-313, 2003.

[76] G. Ferlazzo, M. Tsang, L. Moretta, G. Melioli, R. Steinman, and C. Münz, "Human dendritic cells activate resting natural killer (NK) cells and are recognized via the NKp30 receptor by activated NK cells," Journal of Experimental Medicine, vol. 195, no. 3, pp. 343-351, 2002.

[77] D. Pende, R. Castriconi, P. Romagnani et al., "Expression of the DNAM-1 ligands, Nectin-2 (CD112) and poliovirus receptor (CD155), on dendritic cells: relevance for natural killer-dendritic cell interaction," Blood, vol. 107, no. 5, pp. 2030-2036, 2006.

[78] G. M. Spaggiari, R. Carosio, D. Pende et al., "NK cell-mediated lysis of autologous antigen-presenting cells is triggered by the engagement of the phosphatidylinositol 3-kinase upon ligation of the natural cytotoxicity receptors NKp30 and NKp46," European Journal of Immunology, vol. 31, no. 6, pp. 1656-1665, 2001.

[79] M. Vitale, M. Della Chiesa, S. Carlomagno et al., "NKdependent DC maturation is mediated by TNF-a and IFN-g released upon engagement of the NKp30 triggering receptor," Blood, vol. 106, no. 2, pp. 566-571, 2005.

[80] R. M. Steinman, D. Hawiger, and M. C. Nussenzweig, "Tolerogenic dendritic cells," Annual Review of Immunology, vol. 21, pp. 685-711, 2003.

[81] P. Mantegani, G. Tambussi, L. Galli, C. T. Din, A. Lazzarin, and C. Fortis, "Perturbation of the natural killer cell compartment during primary human immunodeficiency virus 1 infection primarily involving the CD56bright subset," Immunology, vol. 129, no. 2, pp. 220-233, 2010.

[82] A. De Maria, D. Mavilio, P. Costa, P. Dignetti, M. Fogli, and M. C. Mingari, "Multiple HLA-class I-specific inhibitory NK receptor expression and IL-4/IL-5 production by CD8+ Tcell clones in HIV-1 infection," Immunology Letters, vol. 72, no. 3, pp. 179-182, 2000.

[83] D. Mavilio, J. Benjamin, M. Daucher et al., "Natural killer cells in HIV-1 infection: dichotomous effects of viremia on inhibitory and activating receptors and their functional correlates," Proceedings of the National Academy of Sciences of the United States of America, vol. 100, no. 25, pp. 1501115016, 2003.

[84] A. De Maria, A. Ferraris, M. Guastella et al., "Expression of HLA class I-specific inhibitory natural killer cell receptors in HIV-specific cytolytic T lymphocytes: Impairment of specific cytolytic functions," Proceedings of the National Academy of Sciences of the United States of America, vol. 94, no. 19, pp. 10285-10288, 1997.

[85] A. Valentin, M. Rosati, D. J. Patenaude et al., "Persistent HIV1 infection of natural killer cells in patients receiving highly active antiretroviral therapy," Proceedings of the National Academy of Sciences of the United States of America, vol. 99, no. 10, pp. 7015-7020, 2002.

[86] H. B. Bernstein, G. Wang, M. C. Plasterer et al., "CD4+ NK cells can be productively infected with HIV, leading to downregulation of CD4 expression and changes in function," Virology, vol. 387, no. 1, pp. 59-66, 2009.

[87] J. M. Milush, B. R. Long, J. E. Snyder-Cappione et al., "Functionally distinct subsets of human NK cells and monocyte/DC-like cells identified by coexpression of CD56, CD7, and CD4," Blood, vol. 114, no. 23, pp. 4823-4831, 2009.

[88] L. Azzoni, E. Papasavvas, J. Chehimi et al., "Sustained impairment of IFN-gamma secretion in suppressed HIV-infected patients despite mature NK cell recovery: evidence for a defective reconstitution of innate immunity," Journal of Immunology, vol. 168, no. 11, pp. 5764-5770, 2002.

[89] F. Bozzano, M. Nasi, L. Bertoncelli et al., "NK-cell phenotype at interruption underlies widely divergent duration of CD4+guided antiretroviral treatment interruption," International Immunology, vol. 23, no. 2, pp. 109-118, 2011.

[90] G. Alter, M. P. Martin, N. Teigen et al., "Differential natural killer cell-mediated inhibition of HIV-1 replication based on distinct KIR/HLA subtypes," Journal of Experimental Medicine, vol. 204, no. 12, pp. 3027-3036, 2007.

[91] M. P. Martin, X. Gao, J. H. Lee et al., "Epistatic interaction between KIR3DS1 and HLA-B delays the progression to AIDS," Nature Genetics, vol. 31, no. 4, pp. 429-434, 2002. 
[92] A. De Maria and L. Moretta, "NK cell function in HIV-1 infection," Current HIV Research, vol. 6, no. 5, pp. 433-440, 2008.

[93] A. De Maria, R. Biassoni, M. Fogli et al., "Identification, molecular cloning and functional characterization of NKp46 and NKp30 natural cytotoxicity receptors in Macaca fascicularis NK cells," European Journal of Immunology, vol. 31, no. 12, pp. 3546-3556, 2001.

[94] R. Biassoni, M. Fogli, C. Cantoni et al., "Molecular and functional characterization of NKG2D, NKp80, and NKG2C triggering $\mathrm{NK}$ cell receptors in rhesus and cynomolgus macaques: monitoring of NK cell function during simian HIV infection," Journal of Immunology, vol. 174, no. 9, pp. 5695-5705, 2005.

[95] A. De Maria, E. Ugolotti, E. Rutjens et al., "NKp44 expression, phylogenesis and function in non-human primate NK cells," International Immunology, vol. 21, no. 3, pp. 245-255, 2009.

[96] E. Rutjens, S. Mazza, R. Biassoni et al., "Differential NKp30 inducibility in chimpanzee NK cells and conserved NK cell phenotype and function in long-term HIV-1-infected animals," Journal of Immunology, vol. 178, no. 3, pp. 1702-1712, 2007.

[97] J. Corado, F. Toro, H. Rivera, N. E. Bianco, L. Deibis, and J. B. De Sanctis, "Impairment of natural killer (NK) cytotoxic activity in hepatitis C virus (HCV) infection," Clinical and Experimental Immunology, vol. 109, no. 3, pp. 451-457, 1997.

[98] M. G. Lassen, J. R. Lukens, J. S. Dolina, M. G. Brown, and Y. S. Hahn, "Intrahepatic IL-10 maintains NKG2A+Ly49- liver NK cells in a functionally hyporesponsive state," Journal of Immunology, vol. 184, no. 5, pp. 2693-2701, 2010.

[99] B. Amadei, S. Urbani, A. Cazaly et al., "Activation of natural killer cells during acute infection with hepatitis C virus," Gastroenterology, vol. 138, no. 4, pp. 1536-1545, 2010.

[100] S. Pelletier, C. Drouin, N. Bédard, S. I. Khakoo, J. Bruneau, and N. H. Shoukry, "Increased degranulation of natural killer cells during acute HCV correlates with the magnitude of virus-specific T cell responses," Journal of Hepatology, vol. 53, no. 5, pp. 805-816, 2010.

[101] C. Morishima, D. M. Paschal, C. C. Wang et al., "Decreased NK cell frequency in chronic hepatitis $C$ does not affect ex vivo cytolytic killing," Hepatology, vol. 43, no. 3, pp. 573-580, 2006.

[102] P. Bonorino, M. Ramzan, X. Camous et al., "Fine characterization of intrahepatic NK cells expressing natural killer receptors in chronic hepatitis B and C," Journal of Hepatology, vol. 51, no. 3, pp. 458-467, 2009.

[103] U. C. Meier, R. E. Owen, E. Taylor et al., "Shared alterations in NK cell frequency, phenotype, and function in chronic human immunodeficiency virus and hepatitis $\mathrm{C}$ virus infections," Journal of Virology, vol. 79, no. 19, pp. 12365-12374, 2005.

[104] B. Oliviero, S. Varchetta, E. Paudice et al., "Natural killer cell functional dichotomy in chronic hepatitis B and chronic hepatitis C virus infections," Gastroenterology, vol. 137, no. 3, pp. 1151-1160, 2009.

[105] O. Dessouki, Y. Kamiya, H. Nagahama et al., "Chronic hepatitis $\mathrm{C}$ viral infection reduces NK cell frequency and suppresses cytokine secretion: reversion by anti-viral treatment," Biochemical and Biophysical Research Communications, vol. 393, no. 2, pp. 331-337, 2010.

[106] L. Golden-Mason, L. Madrigal-Estebas, E. McGrath et al., "Altered natural killer cell subset distributions in resolved and persistent hepatitis $\mathrm{C}$ virus infection following single source exposure," Gut, vol. 57, no. 8, pp. 1121-1128, 2008.

[107] M. S. Bonavita, A. Franco, M. Paroli et al., "Normalization of depressed natural killer activity after interferon- $\alpha$ therapy is associated with a low frequency of relapse in patients with chronic hepatitis C," International Journal of Tissue Reactions, vol. 15, no. 1, pp. 11-16, 1993.

[108] A. De Maria, M. Fogli, S. Mazza et al., "Increased natural cytotoxicity receptor expression and relevant IL-10 production in NK cells from chronically infected viremic HCV patients," European Journal of Immunology, vol. 37, no. 2, pp. 445-455, 2007.

[109] M. Jinushi, T. Takehara, T. Tatsumi et al., "Negative regulation of NK cell activities by inhibitory receptor CD94/ NKG2A leads to altered NK cell-induced modulation of dendritic cell functions in chronic hepatitis C virus infection," Journal of Immunology, vol. 173, no. 10, pp. 6072-6081, 2004.

[110] T. Takehara and N. Hayashi, "Natural killer cells in hepatitis $\mathrm{C}$ virus infection: from innate immunity to adaptive immunity," Clinical Gastroenterology and Hepatology, vol. 3, no. 10 ,supplement 2, pp. S78-S81, 2005.

[111] G. Ahlenstiel, R. H. Titerence, C. Koh et al., "Natural killer cells are polarized toward cytotoxicity in chronic hepatitis $\mathrm{C}$ in an interferon-alfa-dependent manner," Gastroenterology, vol. 138, no. 1, pp. 325-335, 2010.

[112] R. J. Harrison, A. Ettorre, A. M. Little, and S. I. Khakoo, "Association of NKG2A with treatment for chronic hepatitis $\mathrm{C}$ virus infection," Clinical and Experimental Immunology, vol. 161, no. 2, pp. 306-314, 2010.

[113] J. Nattermann, G. Feldmann, G. Ahlenstiel, B. Langhans, T. Sauerbruch, and U. Spengler, "Surface expression and cytolytic function of natural killer cell receptors is altered in chronic hepatitis C," Gut, vol. 55, no. 6, pp. 869-877, 2006.

[114] F. Bozzano, A. Picciotto, P. Costa et al., "Activating NK cell receptor expression/function (NKp30,NKp46,DNAM-1) during chronic viraemic HCV infection is associated with the outcome of combined treatment," European Journal of Immunology, 2011.

[115] J. C. Yoon, M. Shiina, G. Ahlenstiel, and B. Rehermann, "Natural killer cell function is intact after direct exposure to infectious hepatitis C virions," Hepatology, vol. 49, no. 1, pp. 12-21, 2009.

[116] S. Crotta, M. Brazzoli, D. Piccioli, N. M. Valiante, and A. Wack, "Hepatitis C virions subvert natural killer cell activation to generate a cytokine environment permissive for infection," Journal of Hepatology, vol. 52, no. 2, pp. 183-190, 2010.

[117] S. Knapp, U. Warshow, D. Hegazy et al., "Consistent beneficial effects of killer cell immunoglobulin-like receptor $2 \mathrm{dl} 3$ and group 1 human leukocyte antigen-c following exposure to hepatitis c virus," Hepatology, vol. 51, no. 4, pp. 1168-1175, 2010.

[118] E. Marcenaro, B. Ferranti, M. Falco, L. Moretta, and A. Moretta, "Human NK cells directly recognize Mycobacterium bovis via TLR2 and acquire the ability to kill monocyte-derived DC," International Immunology, vol. 20, no. 9, pp. 1155-1167, 2008.

[119] R. Vankayalapati, B. Wizel, S. E. Weis et al., "The NKp46 receptor contributes to NK cell lysis of mononuclear phagocytes infected with an intracellular bacterium," Journal of Immunology, vol. 168, no. 7, pp. 3451-3457, 2002. 
[120] L. E. Bermudez, M. Wu, and L. S. Young, "Interleukin-12stimulated natural killer cells can activate human macrophages to inhibit growth of Mycobacterium avium," Infection and Immunity, vol. 63, no. 10, pp. 4099-4104, 1995.

[121] K. J. Brill, Q. Li, R. Larkin et al., "Human natural killer cells mediate killing of intracellular Mycobacterium tuberculosis $\mathrm{H} 37 \mathrm{Rv}$ via granule-independent mechanisms," Infection and Immunity, vol. 69, no. 3, pp. 1755-1765, 2001.

[122] P. Katz, H. Yeager Jr., G. Whalen, M. Evans, R. P. Swartz, and J. Roecklein, "Natural killer cell-mediated lysis of Mycobacterium-avium complex-infected monocytes," Journal of Clinical Immunology, vol. 10, no. 1, pp. 71-77, 1990.

[123] M. Lichtner, R. Rossi, F. Mengoni et al., "Circulating dendritic cells and interferon- $\alpha$ production in patients with tuberculosis: Correlation with clinical outcome and treatment response," Clinical and Experimental Immunology, vol. 143, no. 2, pp. 329-337, 2006.

[124] J. Hinchey, S. Lee, B. Y. Jeon et al., "Enhanced priming of adaptive immunity by a proapoptotic mutant of Mycobacterium tuberculosis," Journal of Clinical Investigation, vol. 117, no. 8, pp. 2279-2288, 2007.

[125] R. Vankayalapati, P. Klucar, B. Wizel et al., "NK cells regulate CD8+ T cell effector function in response to an intracellular pathogen," Journal of Immunology, vol. 172, no. 1, pp. 130 137, 2004.

[126] F. Bozzano, P. Costa, G. Passalacqua et al., "Functionally relevant decreases in activatory receptor expression on NK cells are associated with pulmonary tuberculosis in vivo and persist after successful treatment," International Immunology, vol. 21, no. 7, pp. 779-791, 2009. 


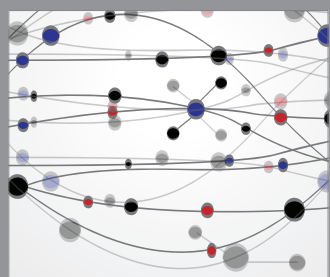

The Scientific World Journal
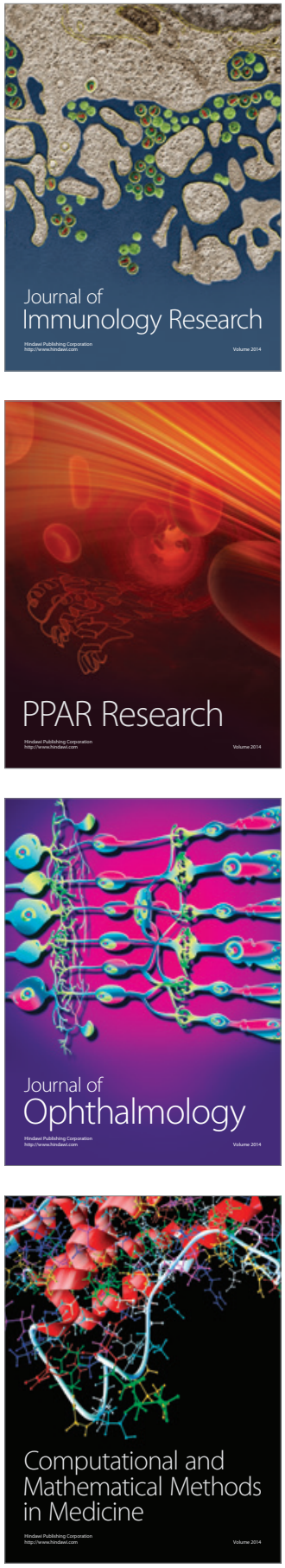

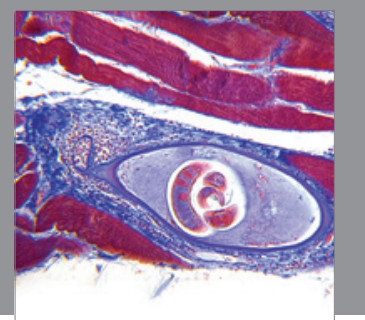

Gastroenterology

Research and Practice
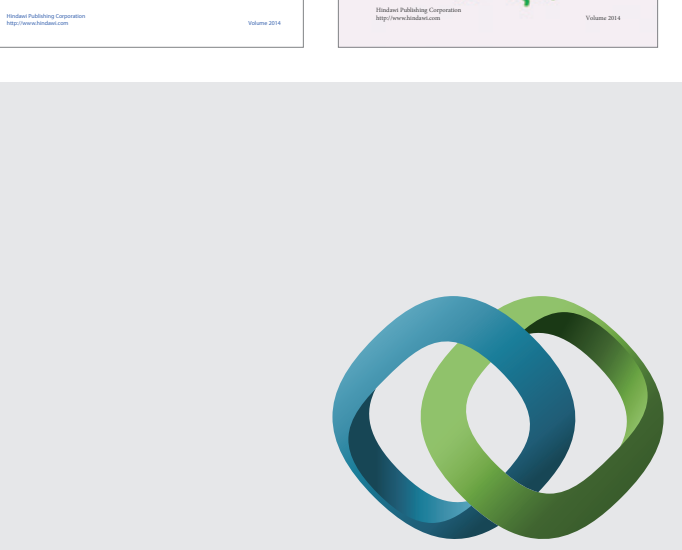

\section{Hindawi}

Submit your manuscripts at

http://www.hindawi.com
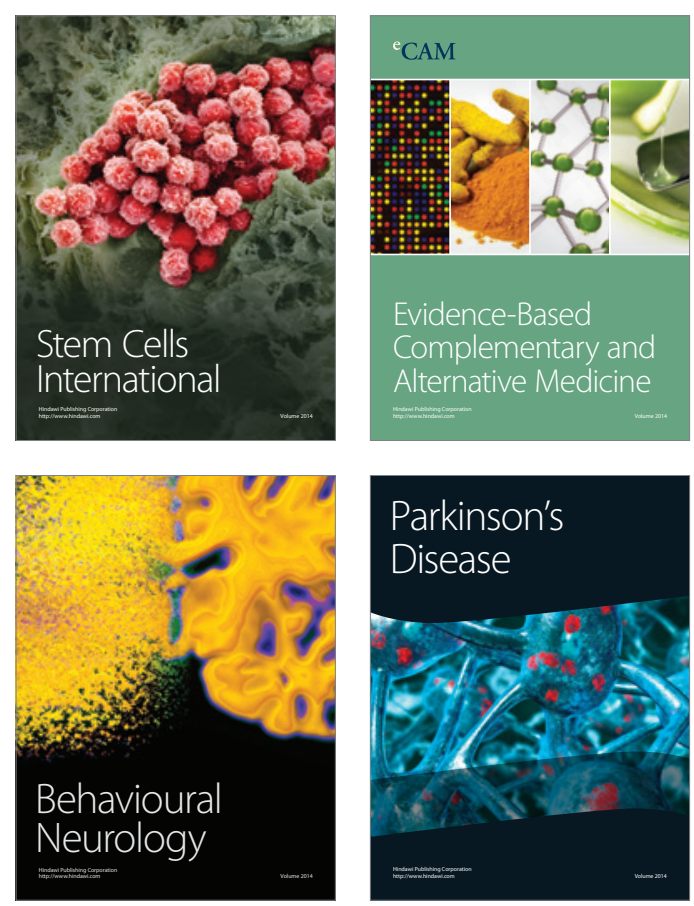

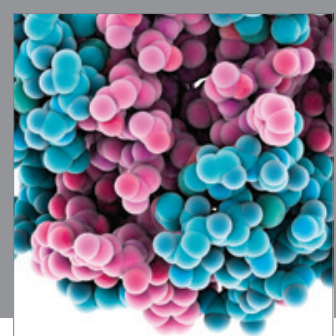

Journal of
Diabetes Research

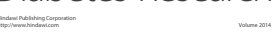

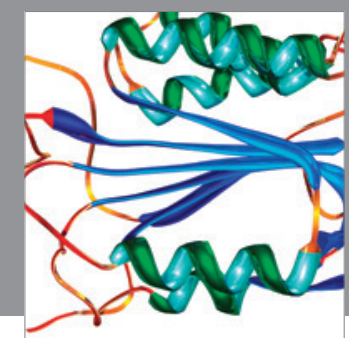

Disease Markers
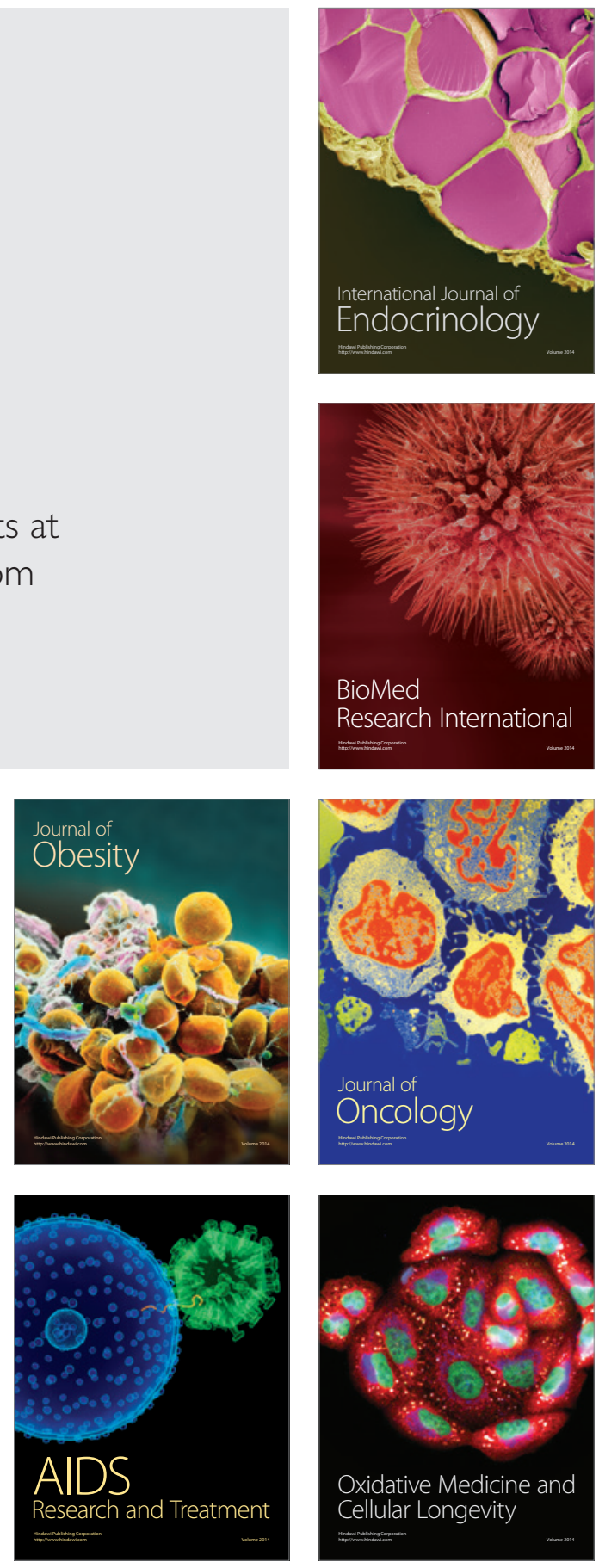